International Mathematical Forum, 1, 2006, no. 27, 1325 - 1334

\title{
On Groups with the Same Set of Order Elements
}

\author{
M.R. Darafsheh and Y. Farjami \\ Department of Mathematics, Statistics and Computer Science \\ Faculty of Science, University of Tehran \\ Tehran, Iran \\ darafsheh@ut.ac.ir
}

\begin{abstract}
A. Sadrudini
Department of Mathematics, Tarbiat Modarres University

P.O. Box 14115-137, Tehran, Iran
\end{abstract}

\begin{abstract}
It is proved that up to isomorphism there are only two finite groups with the same set of element orders as the group $P S L_{6}(3)$.
\end{abstract}

Mathematics Subject Classification: 20D05

Keywords: Element order, Projective Special Linear group

\section{Introduction}

Let $G$ be a finite group. The set of orders of all elements of $G$ is denoted by $\omega(G)$ and is called the spectrum of $G$. This set is closed under divisibility and therefore is uniquely determined by the set $\mu(G)$ of elements in $\omega(G)$ which are maximal under the divisibility relation. By definition a finite group $G$ is called recognizable by the set $\omega(G)$ (in short recognizable) if for every finite group $H$ with $\omega(H)=\omega(G)$ we have $H \cong G$. If there are $k$ non-isomorphic finite groups $H$ for which $\omega(H)=\omega(G)$ then $G$ is called a $k$-recognizable group. A recognizable group is therefore 1 -recognizable.

Up to present time among the linear groups $P S L_{n}(q)$ it is proved that only the groups $P S L_{4}(3)$ and $P S L_{5}(3)$ are recognizable. (See [3] and [7]). But in [8] it is proved that there are infinitely many non-isomorphic groups $G$ such that $\omega(G)=\omega\left(P S L_{3}(3)\right)$. Such groups are called non-recognizable groups. This is because by a result of Shi in [11] every solvable group is a non-recognizable 
group. Now by [8] there is a Frobenius group of the form $K: C$ where $K \cong \mathbb{Z}_{13}$ and $C \cong 2 . S_{4}$ and $\mu(K: C)=\{6,8,13\}=\mu\left(P S L_{3}(3)\right)$.

In this paper we consider the group $P S L_{6}(3)$ and prove.

Main Theorem. Let $G$ be a finite group. Then $\omega(G)=\omega\left(P S L_{6}(3)\right)$ if and only if $G \cong P S L_{6}(3)$ or $P S L_{6}(3):<\theta>$, where $\theta$ is an automorphism of order 2 of $P S L_{6}(3)$.

Throughout the paper we use standard notations. In particular $n$ denotes the cyclic group of order $n$, i.e. $\mathbb{Z}_{n}$, and $A: B$ denotes the semi-direct product of groups $A$ and $B$. For a natural number $n$ the set of prime divisors of $n$ is denoted by $\pi(n)$ and for a finite group $G$ we set $\pi(G)=\pi(|G|)$.

\section{Preliminary results}

For a finite group $G$ the Gruenberg-Kegel graph of $G$ denoted by $G K(G)$ is defined as follows. The vertices of $G K(G)$ are the set of all the prime divisors of $|G|$, and two vertices $p$ and $q$ are adjacent if $G$ contains an element of order $p q$. We denote by $s=s(G)$ the number of connected components of $G K(G)$, and they are denoted by $\pi_{i}=\pi_{i}(G), i \leq 1 \leq s$. If $G$ is of even order then we let $2 \in \pi_{1}$. By $\omega_{i}=\omega_{i}(G)$ and $\mu_{i}=\mu_{i}(G)$ we denote the set of numbers in $\omega(G)$ and $\mu(G)$ respectively all of whose prime divisors belong to $\pi_{i}(G)$. In our study the Gruenberg-Kegel graph plays an important role. If this graph is disconnected, that is $s \geq 2$, then according to the following result quoted in [14] we know the structure of the group in question.

Lemma 1 Let $G$ be a finite group with disconnected Gruenberg-Kegel graph $G K(G)$. Then one of the following holds:

(1) $s(G)=2, G=K H$ is a Frobenius group with kernel $K$ and complement $H$ and the connected components of $G K(G)$ are $\pi(K)$ and $\pi(H)$.

(2) $s(G)=2, G=A B C$, where $A$ and $A B$ are normal subgroups of $G$, $B$ is a normal subgroup of $B C$, and $A B$ and $B C$ are Frobenius groups, with kernels $A$ and $B$ and complements $B$ and $C$ respectively. The two connected components of $G K(G)$ are $\pi(A C)$ and $\pi(B)$. Moreover the groups $B$ and $C$ are cyclic, $|B|$ is odd, and $|C| . \prod_{r \in \pi(A)} r \in \omega(G)$.

(3) There exists a non-abelian simple group $P$ such that $P \unlhd \bar{G}=\frac{G}{K} \unlhd$ Aut $(P)$ for some nilpotent normal $\pi_{1}(G)$-subgroup $K$ of $G$, and $\frac{\bar{G}}{P}$ is a $\pi_{1}(G)$ group. Moreover $G K(P)$ is disconnected, $s(P) \geq s(G)$, and for every $i, 2 \leq$ $i \leq s(G)$, there exists $j, 2 \leq j \leq s(P)$, such that $\omega_{i}(G)=\omega_{j}(P)$.

The finite simple groups $G$ with disconnected $G K(G)$ are classified in [14] and [6]. A list of these groups can be found in [8]. Most of the projective 
special linear groups have connected Gruenberg-Kegel graph. But the ones with $s(G) \geq 2$ are tabulated bellow

Table I

Simple groups $G=P S L_{n}(q)$ with $s(G) \geq 2(p$ is a prime number $)$

\begin{tabular}{|l|l|l|l|l|l|}
\hline$G$ & Condition & $\pi_{1}$ & $\pi_{2}$ & $\pi_{3}$ & $\pi_{4}$ \\
\hline$P S L_{p}(q)$ & $(p, q) \neq(3,2)$, & $\pi\left(q \prod_{i=1}^{p-1}\left(q^{i}-1\right)\right)$ & $\pi\left(\frac{q^{p}-1}{(q-1)(p, q-1)}\right)$ & - & - \\
\hline$P S L_{p+1}(q)$ & $q-1 \mid p+1$ & $\begin{array}{c}\pi\left(q\left(q^{p+1}-1\right)\right. \\
p-1\end{array}$ & $\pi\left(\frac{q^{p}-1}{q-1}\right)$ & - & - \\
& $\prod_{i=1}^{\left.\left(q^{i}-1\right)\right)}$ & & & \\
\hline$P S L_{2}(q)$ & $q \equiv 1(\bmod 4)$ & $\pi(q-1)$ & $\{p\}$ & $\pi\left(\frac{q+1}{2}\right)$ & - \\
\hline$P S L_{2}(q)$ & $q \equiv-1(\bmod 4)$ & $\pi(q+1)$ & $\{p\}$ & $\pi\left(\frac{q-1}{2}\right)$ & - \\
\hline$P S L_{2}(q)$ & $q>2, q$ even & $\{2\}$ & $\pi(q-1)$ & $\pi(q+1)$ & - \\
\hline$P S L_{3}(4)$ & - & $\{2\}$ & $\{3\}$ & $\{5\}$ & $\{7\}$ \\
\hline
\end{tabular}

Therefore if $q=3$ and $p$ is an odd prime number then we have $s\left(P S L_{n}(3)\right)=$ 2 if $n=p$ or $p+1$. The connected comments of the Gruenberg-Kegel graph of $P S L_{n}(3)$ are $\pi_{1}=\pi\left(3 \prod_{i=1}^{p-1}\left(3^{i}-1\right)\right)$ or $\pi_{1}=\pi\left(3\left(3^{p+1}-1\right) \prod_{i=1}^{p-1}\left(3^{i}-1\right)\right)$ in the respective cases $n=p$ or $p+1$. In any case the second component is $\pi_{2}=\pi\left(\frac{3^{p}-1}{2}\right)$.

The group $P S L_{6}(3)$ has order $2^{11} \cdot 3^{15} \cdot 5.7 .11^{2} \cdot 13^{2}$ and its components are $\pi_{1}=\{2,3,5,7,13\}$ and $\pi_{2}=\{11\}$. To find the set of orders of elements of $P S L_{6}(3)$ we use [2].

Lemma 2 Let $G=P S L_{6}(3)$. Then

$$
\begin{aligned}
\mu(G)= & \{36,78,80,104,120,121,182\} \\
\omega(G)= & \{1,2,3,4,5,6,7,8,9,10,11,12,13,14,15,16,18,20,24,26,30 \\
& 36,39,40,52,60,80,91,104,120,121,182\} .
\end{aligned}
$$

According to Lemma 2 the graph $G K\left(P S L_{6}(3)\right)$ may be drawn as follows: 
Lemma 3 If $G$ is a simple group with $\{2,11\} \subseteq \pi(G) \subseteq\{2,3,5,7,11,13\}$ then $G$ is isomorphic to one of the following groups

(1) $\mathbb{A}_{n}, 11 \leq n \leq 16$

(2) $M_{11}, M_{12}, M^{c} L, F i_{22}, H S, S u z, M_{22}$,

(3) $P S L_{2}(11), P S L_{5}(3), P S L_{6}(3), P S U_{5}\left(2^{2}\right), P S U_{6}\left(2^{2}\right)$.

Proof. Clearly $G$ is a non-abelian simple group. According to the classification of the finite simple groups we consider the following cases:

Case $1 G$ is isomorphic to an alternating group. Since 11||$G \mid$ but $7 \nmid|G|$ we must have $G \cong \mathbb{A}_{n}, 11 \leq n \leq 16$.

Case $2 G$ is isomorphic to a sporadic simple group. In this case by inspecting the list of sporadic simple groups in [1] the possible groups listed above are obtained.

Case $3 G$ is isomorphic to a simple group of Lie type. We use the list of orders of these groups given in [1]. If $G$ is defined over a field with characteristic $p$, then since $p|| G \mid$ we must have $p=2,3,5,7,11$ or 13 . Using the fact that the orders of $G$ is divisible by numbers of the form $p^{k} \pm 1$ and $11 \in \pi(G)$ we continue as follows.

If $p=2$, then the order of 2 modulo 11 is 10 and we have $11 \mid 2^{5}+1$. Since $2^{7}-1=127$ is a prime not in $\pi(G)$ we must have $G \cong P S U_{5}\left(2^{2}\right)$ or $P S U_{6}\left(2^{2}\right)$.

If $p=3$, then $11 \mid 3^{5}-1$, hence $G \cong P S L_{5}(3)$ or $P S L_{6}(3)$.

If $p=5$, then $11 \mid 5^{5}-1$, but $5^{3}-1=4 \times 31$, and since $31 \notin \pi(G)$ we don't obtain a possibility.

If $p=7$, then $11 \mid 7^{5}+1$. Since $7^{3}+1=8 \times 43$ and $43 \notin \pi(G)$ there is no possibility in this case as well.

If $p=11$, then $G$ is defined over the Galois field $G F(11)$ and since $11^{2}+$ $1=2 \times 61$ and $11^{3}-1=70 \times 19$ and $61,19 \notin \pi(G)$ the only possibility is $G \cong P S L_{2}(11)$.

If $p=13$, then $11 \mid 13^{5}+1$ and since $13^{4}-1=1680 \times 17$ and $17 \notin \pi(G)$ we don't obtain a possibility. Therefore all the cases are examined and the Lemma is proved now.

Lemma 4 If $G$ is a finite group and $\omega(G)=\omega\left(P S L_{6}(3)\right)$, then $G$ is a nonsolvable group.

Proof. Suppose $G$ is a solvable group. Let $H$ be a $\{5,7,11\}-$ Hall subgroup of $G$. By Lemma 2 the group $G$ does not contain elements of order 5.7, 5.11 and 7.11. Hence by Theorem 1 in $[5]$ we must have $|\pi(G)| \leq 2$, a contradiction. The Lemma is proved now. 


\section{Proof of the Main Theorem}

Theorem 5 If $G$ is a finite group such that $\omega(G)=\omega\left(P S L_{6}(3)\right)$, then $G \cong P S L_{6}(3)$ or $P S L_{6}(3):<\theta>$, where $\theta$ is the graph automorphism of $P S L_{6}(3)$.

Proof. By Lemma 2 the graph $G K(G)$ has two connected components $\pi_{1}=\{2,3,5,7,13\}$ and $\pi_{2}=\{11\}$. Therefore by Lemma 1 we have one of the following three cases that we are going to deal with in separate Lemmas:

Lemma 6 Case 1 is impossible.

Proof. If case 1 holds then $G=K H$ is a Frobenius group with kernel $K$ and complement $H$ and the connected components of $G K(G)$ are $\omega(K)$ and $\omega(H)$. By a famous theorem of Thompson $K$ is nilpotent. If $H$ is a solvable group, then $G$ will be solvable which is not the case by Lemma 4 . Therefore $H$ is a non-solvable Frobenius complement. Now by [10] the group $H$ contains a normal subgroup $H_{0}$ such that $\left[H: H_{0}\right] \leq 2$ and $H_{0} \cong Z \times S L_{2}(5)$ where every Sylow subgroup of $Z$ is cyclic and $\pi(Z) \cap\{2,3,5\}=\varnothing$. Since the group $G$ does not contain elements of order 5.7 and 5.11 , therefore $\{7,11\} \subseteq \pi(K)$. Now being a nilpotent group, $K$ must contain an element of order 77 which contradicts Lemma 2. Hence case 1 does not hold and the Lemma is proved.

\section{Lemma 7 Case 2 is impossible.}

Proof. If case 2 holds, then $G=A B C$, where $A$ and $A B$ are normal subgroups of $G, B$ is a normal subgroup of $B C, B$ and $C$ are cyclic groups, and $A B$ and $B C$ are Frobenius groups with kernels $A$ and $B$ and complements $B$ and $C$ respectively. The connected components of $G K(G)$ are $\omega(B)$ and $\omega(A C)$. Since $A$ and $B$ are Frobenius kernels hence they are nilpotent. Therefore the group $A B$ is solvable. Since $A B \unlhd G$ and $C$ is also solvable we deduce that $G$ is also solvable which violates Lemma 4 . Therefore case 2 is impossible as claimed.

Thus case 3 holds, that is there exists a non-abelian simple group $P$ such that $P \unlhd \bar{G}=\frac{G}{K} \unlhd A u t(P)$ for some nilpotent normal $\pi_{1}(G)$-subgroup $K$ of $G$, and $\frac{\bar{G}}{P}$ is a $\pi_{1}(G)$-group. Moreover $G K(P)$ is disconnected, $s(P) \geq s(G)$, and for every $i, 2 \leq i \leq s(G)$, there exists $j, 2 \leq j \leq s(P)$, such that $\omega_{i}(G)=\omega_{j}(P)$. Since $s(G)=2$ we obtain $\omega_{2}(P)=\omega_{2}(G)=\{11\}$. Obviously $\pi(P) \subseteq \pi(G)$ and since $P$ is a non-abelian simple group we have $\{2,11\} \subseteq$ $\pi(P) \subseteq\{2,3,7,11,13\}$. By Lemma 3 one of the following possibilities occur for $P$ that we are going to deal with them separately: $\mathbb{A}_{11}, \mathbb{A}_{12}, \mathbb{A}_{13}, \mathbb{A}_{14}$, $\mathbb{A}_{15}, \mathbb{A}_{16}, M_{11}, M_{12}, M^{c} l, F i_{22}, H S, S u z, M_{22}, P S L_{2}(11), P S L_{5}(3), P S L_{6}(3)$, $P S U_{5}\left(2^{2}\right), P S U_{6}\left(2^{2}\right)$. 
But before continuing our case by case analysis we will mention a useful result taken from $[9]$.

Lemma 8 Let $H$ be a finite group, $K \unlhd H$ and $\frac{H}{K}$ be a Frobenius group with kernel $F$ and cyclic complement $C$. If $(|F|,|K|)=1$ and $F$ is not contained in $\frac{K C_{H}(K)}{K}$, then $p|C| \in \omega(H)$ for some prime divisor $p$ of $|K|$.

Lemma 9 The group $P S L_{n}(q)$ contains a Frobenius group with kernel an abelian group of order $q^{n-1}$ and complement a cyclic group of order $\frac{q^{n-1}-1}{(n, q-1)}$

Proof. Let $H=<A>, A \in G L_{n-1}(q), O(A)=q^{n-1}-1$, and set $L=\left\{\left[\begin{array}{c|c}(\operatorname{det} X)^{-1} & d_{1} \cdots d_{n-1} \\ \hline 0 & X\end{array}\right] \mid X \in H, a_{i} \in G F(q), 1 \leq i \leq n-1\right\} \leq S L_{n}(q)$.

Then $L$ is a Frobenius group with kernel $S=\left\{\left[\begin{array}{c|c}1 & a_{1} \cdots a_{n-1} \\ \hline 0 & I\end{array}\right] \mid a_{i} \in G F(q), 1 \leq\right.$ $i \leq n-1\}$ of order $q^{n-1}$ and complement $T=\left\{\left[\begin{array}{c|c}(\operatorname{det} X)^{-1} & 0 \\ \hline 0 & X\end{array}\right] \mid X \in H\right\}$ of order $q^{n-1}-1$. Obviously $S$ is abelian and $T$ is cyclic. Let $Z$ denote the centre of $S L_{n}(q)$. The image of $L=S T$ in $P S L_{n}(q)$ is the group $\bar{L}=\frac{Z S T}{Z}=$ $\frac{(Z S)(Z T)}{Z} \cong\left(\frac{Z S}{Z}\right)\left(\frac{Z T}{Z}\right)=\bar{S} \bar{T}$, where $\bar{S} \cong S$ and $\bar{T} \cong \frac{T}{Z}$ and $\bar{L}$ is a Frobenius group with kernel $\bar{S}$ and complement $\bar{T}$.

Next we start the examination of each possibilities for the simple group $P$ with the conditions stated above. From $\bar{G}=\frac{G}{K}$ we know that $\mu(\bar{G}) \subseteq \mu(G)$ and $\omega(\bar{G}) \subseteq \omega(G)$. For the above simple groups $P$ we use [1] for the inspection of the set $\mu(P)$.

Assume $P \cong \mathbb{A}_{n}, 11 \leq n \leq 16$. Since these groups contain elements of order 35 and by Lemma $235 \notin \omega(G)$, hence these possibilities for $P$ lead to a contradiction.

Suppose $P \cong M_{11}, M_{12}, M^{c} l, H S, M_{22}, P S L_{2}(11), P S U_{5}\left(2^{2}\right)$, or $P S L_{5}\left(2^{2}\right)$. By [1] we have $\operatorname{Aut}(P)=P: S$ where the order of $S$ is at most 6 . Therefore $\bar{G} \cong \frac{G}{K}=P: A$ where $|A| \mid 6$. We may assume $K$ is an elementary abelian $p-$ group for some $p \in \pi_{1}(G)$. For the above groups by [1] we have $13 \nmid|P|$, hence 13||$K \mid$. Again by [1] all the above groups contain a group isomorphic to the linear groups $P S L_{2}(11)$. But $P S L_{2}(11)$ has a Frobenius group $\frac{H}{K}$ with kernel $F \cong \mathbb{Z}_{11}$ and complement $C \cong \mathbb{Z}_{5}$. Since $\frac{K C_{H}(K)}{K} \cong \frac{C_{H}(K)}{K \cap C_{H}(K)}$ and by Lemma 2 the group $G$ contains no element of order 11.13 we deduce that $F$ is not contained in $\frac{K C_{H}(K)}{K}$, hence all the conditions of Lemma 8 are fulfilled. Therefore $13.5=$ $65 \in \omega(H)$ contradicting Lemma 2. In this way the above possibilities are ruled out. 
Next suppose $P \cong F i_{22}$ or $S u z$. In both cases by [1] we observe that $P$ contains an element of order 21 which violates Lemma 2 .

Now let $P \cong P S L_{5}(3)$. In this case $\operatorname{Aut}(P)=P: 2$ and from the order of $P$ we deduce that 7||$K \mid$. By [4] the group $P S L_{5}(3)$ contains a group isomorphic to $M_{11}$, hence it contains a copy of the group $P S L_{2}(11)$. Now again we consider the Frobenius subgroup $\mathbb{Z}_{11}: \mathbb{Z}_{5}$ and drive a contradiction.

Finally we are left with $P \cong P S L_{6}(3)$. As we mentioned above by [13] we may assume that $K$ is an elementary abelian $p$-group for some $p \in \pi_{1}(G)=$ $\{2,3,5,7,13\}$ and $K$ is a module for $P$ on which $P$ acts faithfully and $C_{G}(K)=$ $K$. Our first aim is to prove $K=1$ is the identity group. Let us consider the Frobenius subgroup of $P S L_{6}(3)$ of the shape $3^{5}: \frac{3^{5}-1}{(6,3-1)}=3^{5}: 121$ which is constructed in Lemma 9. If $p \neq 3$, then by Lemma 8 the group $G$ must contain an element of order $121 p$ which is not possible by Lemma 2 . Therefore we may assume $p=3$. Let $|K|=3^{k}$. Since $7 \in \omega(P)$ and the order of 7 modulo 2 is 6 , hence $6 \mid k$. We put $K=3^{k}, k \geq 1$, an elementary abelian 3-group. In this case $\frac{G}{K}$ is isomorphic to a subgroup of $G L_{k}(3)$. But $\frac{G}{K}$ also contains a group isomorphic to $P \cong P S L_{6}(3)$, hence $3^{k} \cdot P S L_{6}(3) \leq G \leq 3^{k} \cdot G L_{k}(3)$. Now if a subgroup $H$ of $P S L_{6}(3)$ splits over $3^{k}$, then $3^{k}: H$ would be a subgroup of the affine group of the vector space of dimension $k+1$ over $\mathbb{Z}_{3}$ and the elements of the group $3^{k}: H$ would be of the form $\left[\begin{array}{ll}1 & v \\ 0 & A\end{array}\right]$ in the group $G L_{k+1}(3)$.

Our notation is chosen so that $\bar{A}=Z A$, where $Z=\{ \pm I\}$ is the centre of $S L_{6}(3)$ and $A \in S L_{6}(3)$. If $X=\left[\begin{array}{ll}1 & v \\ 0 & A\end{array}\right]$, then it is easy to show that for all $k \in \mathbb{N}$ we have

$$
X^{k}=\left[\begin{array}{cc}
1 & v+v \bar{A}+\cdots+v(\bar{A})^{k-1} \\
0 & (\bar{A})^{k}
\end{array}\right]
$$

where $v \bar{A}=v A$ and so on. Therefore if $O(\bar{A})=n$ and $v$ is chosen so that $u=v+v \bar{A}+\cdots+v(\bar{A})^{n-1} \neq 0$, then the order of $X=\left[\begin{array}{ll}1 & v \\ 0 & \bar{A}\end{array}\right]$ is $3 n$.

Now the following matrix

$$
A=\left[\begin{array}{rrrrrr}
-1 & 0 & 0 & 0 & 0 & 0 \\
0 & 1 & 0 & 0 & 0 & 0 \\
0 & 0 & 0 & 1 & 0 & 0 \\
0 & 0 & 0 & 0 & 1 & 0 \\
0 & 0 & 0 & 0 & 0 & 1 \\
0 & 0 & 1 & 0 & -1 & 0
\end{array}\right]
$$

is in $S L_{6}(3)$ and $O(\bar{A})=16$. Moreover $\langle\bar{A}\rangle$ is a subgroup of order 16 in $P S L_{6}(3)$ which splits over $3^{k}$. By direct calculation we see that $I+\bar{A}+(\bar{A})^{2}+$ 
$\cdots+(\bar{A})^{15} \neq 0$, hence the order of $X=\left[\begin{array}{ll}1 & v \\ 0 & \bar{A}\end{array}\right]$, where $v=(1,0,0,0,0,0)$, as an element of $3^{6} \cdot P S L_{6}(3)$ is 48 , contradicting Lemma 2. Hence $K=1$ as we claimed. Therefore we have $P \leq G \leq A u t(P)$ where $P \cong P S L_{6}(3)$. By [12] every automorphism of the group $P S L_{n}(q)$ is a product of inner, field, graph and diagonal automorphism of the group. Let $q=p^{f}, p$ prime, and let $\sigma_{p}$ denote the Frobenius automorphism of the Galois field $G F(q)$. Then $\sigma_{p}$ induces an automorphism of order $f$ of the group $P S L_{n}(q)$ in the natural way. This automorphism is denoted by $\sigma_{p}$ again and is called a field automorphism of $P S L_{n}(q)$. If $A \in G L_{n}(q)$, then $\delta=\delta_{A}$ defined by $\delta_{A}(B)=A^{-1} B A, \forall$ $B \in S L_{n}(q)$, is an automorphism of $S L_{n}(q)$ and induces an automorphism of $S L_{n}(q)$ in the usual way which is denoted by $\delta_{A}$ again. The automorphisms of $P S L_{n}(q)$ of the above type are called diagonal automorphisms and it is known that they form a group whose order modulo $P S L_{n}(q)$ is $d=(n, q-1)$. Finally if $n \geq 3$, then the mapping defined by $\theta(A)=\left(A^{-1}\right)^{t}, A \in S L_{n}(q)$, is an outer automorphism of $S L_{n}(q)$ of order 2 which induces an outer automorphism of $P S L_{n}(q)$ which is denoted by $\theta$ again and is called the graph automorphism of $P S L_{n}(q)$.

Now in the case of $P \cong P S L_{6}(3)$ we have $\operatorname{Aut}(P)=P: S$ where $S=<\delta, \theta>$ is generated by a diagonal automorphism $\delta$ and the graph automorphism $\theta$. We have $\delta^{2}=\theta^{2}=1, \delta \theta=\theta \delta$, hence $S$ is isomorphic to the Klein fourgroup. In this case we define $\delta=\delta_{A}$, where $A=\operatorname{diag}(-1,1,1,1,1,1)$ the diagonal matrix with -1 and 1 along the main diagonal. Therefore for $G$ we have the following possibilities: $P, P:\langle\theta\rangle, P:\langle\delta\rangle, P:\langle\theta \delta\rangle, P:\langle\theta, \delta\rangle$.

Assume $G=P:<\delta>$. First we consider the group $S L_{6}(3):<\delta>$. Then using $\delta=\delta_{A}$ with $A=\operatorname{diag}(-1,1,1,1,1,1)$ we obtain $C_{S L_{6}(3)}(\delta)=$ $\left\{\left[\begin{array}{ll}a & 0 \\ 0 & A\end{array}\right] \mid a \operatorname{det}(A)=1, A \in G L_{5}(3)\right\}$. Now the group $S L_{5}(3)$ has an element of order 121 and from the above we obtain an element of order 242 in $S L_{6}(3):<$ $\delta>$ and consequently in $P S L_{6}(3):<\delta>$, contradicting Lemma 2 again.

Next we will assume $G=P:<\theta \delta>$. If $A \in S L_{6}(3)$, then using the action of $\theta$ we obtain $(A \theta \delta)^{2}=A \theta \delta A \theta \delta=A \delta \theta A \theta \delta=A \delta\left(A^{-1}\right)^{t} \delta$. Now we choose $A=\left[\begin{array}{ll}\tau & 0 \\ 0 & \eta\end{array}\right]$ a block diagonal matrix such that $\tau \in S L_{2}(3)$ and $\eta \in S L_{4}(3)$. Using $\delta=\operatorname{diag}(-1,1,1,1,1,1)$ we obtain $A \delta\left(A^{-1}\right)^{t} \delta=\left[\begin{array}{cc}\tau \alpha\left(\tau^{-1}\right)^{t} \alpha & 0 \\ 0 & \eta\left(\eta^{-1}\right) t\end{array}\right]$ where $\alpha=\left[\begin{array}{cc}-1 & 0 \\ 0 & 1\end{array}\right]$. Using [1] we see that the group $S L_{4}(3):\langle\theta\rangle$, where $\theta$ is again the graph automorphism of $S L_{4}(3)$, has an element $\eta \theta$ of order 36 . Therefore $(\eta \theta)^{2}=\eta \theta \eta \theta=\eta\left(\eta^{-1}\right)^{t}$ has order 18. Now we chose $\tau=\left[\begin{array}{ll}1 & 1 \\ 0 & 1\end{array}\right]$ 
$\in S L_{2}(3)$ and calculate $\tau \alpha\left(\tau^{-1}\right)^{t} \alpha=\left[\begin{array}{cc}-1 & 1 \\ 1 & 1\end{array}\right]$ which is a matrix of order 4 . Therefore $(A \theta \delta)^{2}$ has order 36 and consequently $A \theta \delta$ is an element of order 72 in $S L_{6}(3):<\theta \delta>$. The image of $A \theta \delta$ in $P S L_{6}(3):<\theta \delta>$ also has order 72 which is against Lemma 2 .

Since $P:<\theta, \delta>$ contains the group $P:<\delta>$, hence it is not a possibility.

Finally we are left with $G=P \cong P S L_{6}(3)$ or $G=P:<\theta>$. But if $G=P:<\theta>$, then $G=P \cup P \theta$, and if we set $Z=Z\left(S L_{6}(3)\right)=\{ \pm I\}$, then for $Z A \theta \in P \theta$, we have $(Z A \theta)^{2}=Z A\left(A^{-1}\right)^{t}$, where $A \in S L_{6}(3)$. Therefore order of $Z A \theta$ is twice the order of $Z A\left(A^{-1}\right)$. But using a computer program we found the order of all matrices of the forms $A(A)^{-1}$, where $A \in S L_{6}(3)$, and found that $O(Z A \theta) \in \omega(G)$. Therefore $\omega(P)=\omega(P:<\theta>)$ and the theorem is proved.

\section{Acknowledgement}

The first author would like to thank the research council of the University of Tehran for financial support.

\section{References}

[1] J. H. Conway, R. T. Curtis, S. P. Norton, R. A. Parker and R. A. Wilson, Atlas of finite groups, Clarendon Press, Oxford, 1985.

[2] M. R. Darafsheh and Y. Farjami, Computing the order of elements in the finite linear groups, submitted.

[3] M. R. Darafsheh and A. R. Moghaddamfar, A characterization of some finite groups by their element orders, Algebra Colloq., 7 (2000), 467-476.

[4] L. Di Martino and A. Wagner, The irreducible subgroups of $\operatorname{PSL}\left(V_{5}, q\right)$ where $q$ is odd, Result. Math., 2 (1979), 54-61.

[5] G. Higman, Finite groups in which every element has prime power order, J. London Math. Soc., 32 (1957), 335-342.

[6] A. S. Kondratév, On prime graph components of finite simple groups, Mat. Sb., 180 (1989), 787-797.

[7] S. Lipschutz and W. J. Shi, Finite groups whose element orders do not exceed twenty, Progr. Nat. Sc., 10 (2000), 11-21.

[8] V. D. Mazurov, Recognition of finite simple groups $S_{4}(q)$ by their element orders, Algebra and Logic, 41 (2002), 93-110.

[9] V. D. Mazurov, Characterization of finite groups by sets of orders of their elements, Algebra and Logic, 36 (1997), 23-32. 
[10] D. S. Passman, Permutation groups, W. A. Benjamin Inc., New York, 1968.

[11] W. J. Shi, Groups whose elements have given orders, Chin. Sc. Bull., 42 (1997), 1761-1764.

[12] R. Steinberg, Automorphisms of finite linear groups, Canad. J. Math., 12 (1960), 606-615.

[13] A. V. Vasilyev, Recognizing groups $G_{2}\left(3^{n}\right)$ by their element orders, Algebra and Logic, 41 (2002), 74-80.

[14] J. S. Williams, Prime graph components of finite groups, J. Algebra, 69 (1981), 487-513.

Received: November 5, 2005 